\title{
Laparoscopic resection of adult small bowel intussuscepting lipoma with intracorporeal anastomosis: a case report
}

\begin{abstract}
GI intussusception, although relatively common in pediatric populations, is a unique and infrequent nidus for adult morbidity. The case presented is of a 41 -year-old female initially complaining of smoldering vague abdominal pain with acute brief episodes of worsening crampy pain. She was found to have an intussuscepting small bowel on computed tomography $(\mathrm{CT})$ scan and opted to have diagnostic laparoscopy. The procedure was performed entirely intracorporeally and included a small bowel resection of the patient's mid-jejunum with accompanying re-anastamosis. Pathology showed a resected segment of jejunum, $3.8 \mathrm{~cm}$ in length $\times 2.0 \mathrm{~cm}$ in diameter. The specimen contained a freely mobile intraluminal mass $3.0 \times 1.6 \times 1.3 \mathrm{~cm}$, tan color, adipocytic, and well-circumscribed consistent with small bowel lipoma on histology, which had arisen from a $2 \mathrm{~cm}$ bowel wall defect and was partially covered by serosa. The patient had appropriate gastrointestinal symptom relief after the procedure while in-house and she endorsed complete resolution of abdominal symptoms at her 2 week and 6month follow-up appointments.
\end{abstract}

Keywords: adult, small bowel, intussusceptions, lipoma, laparoscopic
Volume 9 Issue I - 2018

Scott Rupp, Scott Whitecar, Peter $\mathrm{Ng}$, Alexander Malloy, Kevin Lin Hurtubise Department of Surgery/General Surgery Service, Tripler Army Medical Center, USA

Correspondence: Scott Rupp, Department of Surgery/ General Surgery Service, Tripler Army Medical Center, USA, Email scott.rupp@usuhs.edu

Received: November 22, 2017 | Published: February 01, 2018

\section{Introduction}

Intussusception, derived from the Latin roots intu "within" and susceptio "action of undertaking," is the invagination of segments of bowel creating an often-transient obstruction. Adult cases account for only $5 \% .{ }^{1}$ of all gastrointestinal intussusception cases and are commonly attributed to the formation of a small bowel lipoma. Adult intussusception is a very rare occurrence with some estimates of only 2 cases per year in the United States. ${ }^{2}$ Intussusception can further be classified by the location along gastrointestinal tract: enteroenteric, colocolic, ileocolic, and ileocecal. ${ }^{3}$ Although there is no specific sign of intussusception in adults, abdominal pain is frequently investigated with CT with oral and intravenous (IV) contrast imaging which can reveal a telescoped segment of bowel, "target sign."

\section{Case details}

The patient initially presented to the emergency room at Tripler Army Medical Center with complaints of acute onset right lower quadrant abdominal pain that radiated to her back. Physicians were concerned for appendicitis and ordered a CT abdomen and pelvis scan which revealed telescoping of the small bowel secondary to a possible $1.9 \mathrm{~cm}$ mid-jejunal lipoma (lead point) without clinical or radiographic evidence of obstruction. She was discharged home as her symptoms remitted and was referred to the general surgery clinic. When seen in clinic, she exhibited continuing low intensity abdominal pain that was non-radiating, and located just inferior and to the right of her umbilicus. She stated that the pain was exacerbated by palpation and is occasionally associated with transient nausea. Her back pain has resolved and she later contributed this pain to an injury sustained during jiu-jitsu training. The patient has not tried taking any medication for the back or abdominal pain and does not complain of any associated concerns with her bowel movements or gastrointestinal habits. She denies any vomiting, diarrhea, chest pain, shortness of breath, hematochezia, or melena.
The surgery team expressed concern regarding a probable intraluminal mass such as a small bowel lipoma causing the intussusception with potential for impending bowel obstruction or hemorrhage. She was therefore recommended for surgery due to the aforementioned reasons and also due to her chronicity of intermittent crampy abdominal pain and increasing anxiety. A diagnostic laparoscopy with likely small bowel resection was scheduled and the patient was counseled regarding the risks, benefits, and alternative means of treatment with verbal understanding. The patient's only reported past medical history was of ovarian cysts as a teen. Her previous surgeries included breast augmentation, left knee arthroplasty, and bilateral salpingectomy. Her only significant family medical history was of a mother who died of pancreatitis.

Her physical exam was notable for mild right lower quadrant abdominal tenderness without guarding and periumbilical tenderness without evidence of a palpable mass. Laboratory results were unremarkable with no elevated white blood cell (WBC) count, abnormalities in differentiated complete blood count, or elevated lactate. Her $\mathrm{CT}$ abdomen and pelvis with IV and oral contrast revealed an intraluminal oval homogenous mass measuring $1.9 \times 1.1 \mathrm{~cm}$ in the left hemiabdomen within the mid-jejunum (Figure 1).

The small bowel lipoma was isolated on the jejunum and observed in active intussusception (Figure 2). The segment of intussuscepted jejunum was resected and re-anastomosed (Figure 3) without complications. The excised specimen was examined extracorporeally (Figure 4) before being sent for tissue evaluation by pathology. The gross specimen was consistent with $\mathrm{CT}$ imaging and revealed a $2 \mathrm{~cm}$ subserosal lipoma. The resected bowel was incised on the back table to reveal an intraluminal lipoma adherent to the jejunal wall without evidence of necrosis or hemorrhage. Pathologic examination confirmed the diagnosis of a homogenous, subserosal lipoma of the jejunum (Figure 5). 


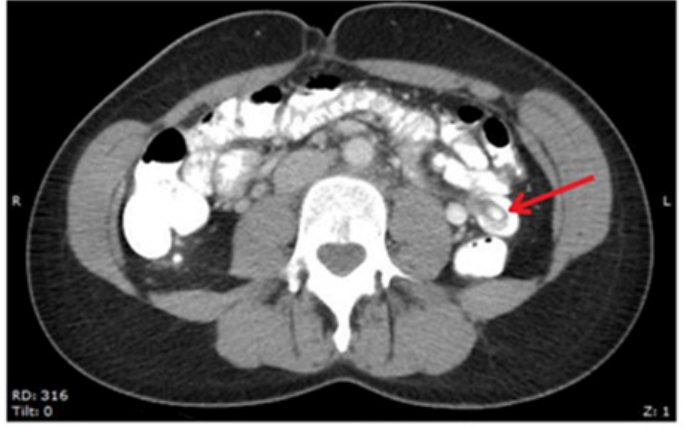

Figure I Radiographic (CT) image of intussusceptions Radiographic”targetsign”.

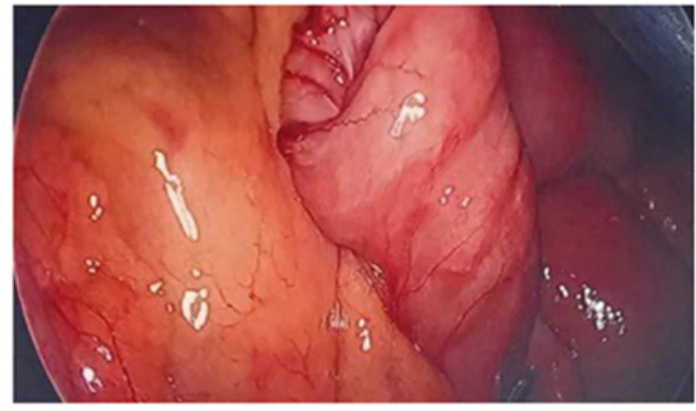

Figure 2 Small bowel in active intussusceptions.

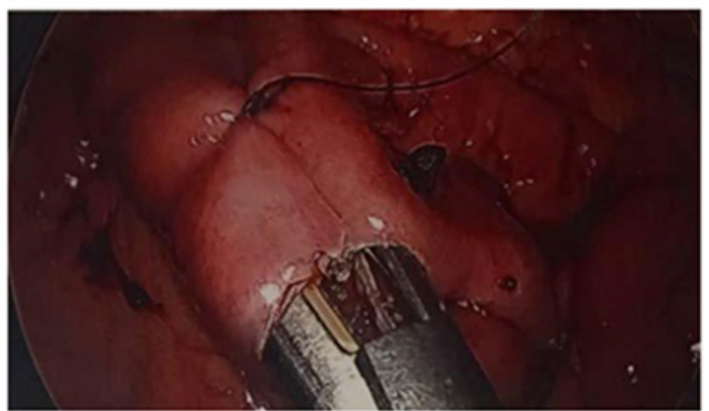

Figure 3 Intracorporeal anastomosis of small bowel.

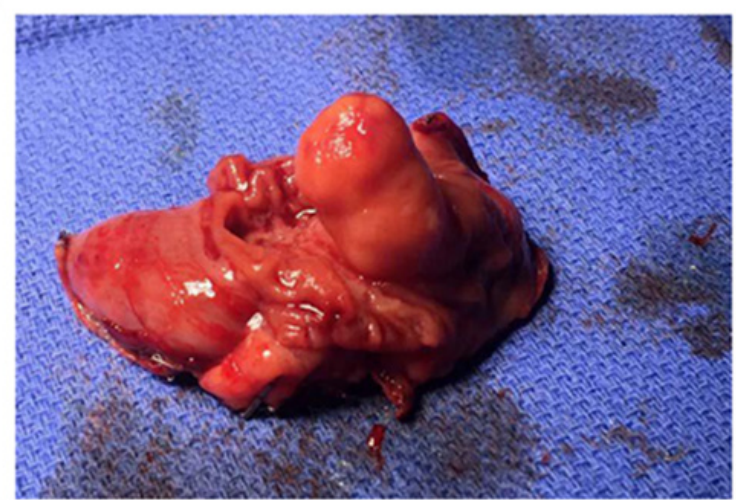

Figure 4 Intaluminal view of resected gross specimen.

The patient had an unremarkable post-operative recovery with return to normal diet and bowel function in three days. She was discharged on the third day with a two-week follow-up appointment, and was doing well at that time and also at subsequent follow-up 6 months later.

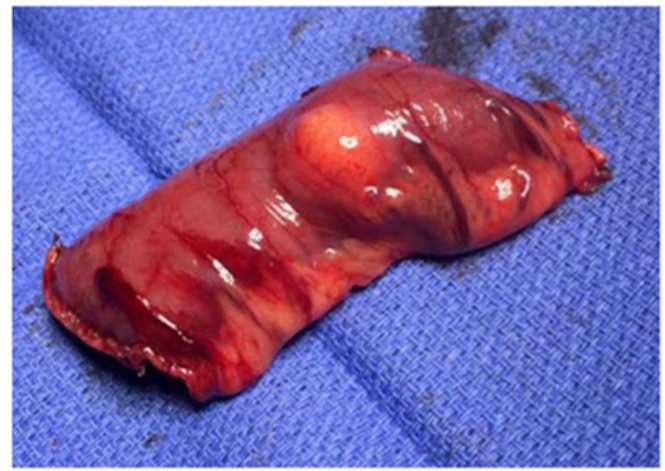

Figure 5 Subserosal lipoma, gross specimen.

\section{Discussion}

Lipomas, benign tumors of adipose tissue, are the most common soft tissue tumors that arise during adulthood, most often between the ages of 40 and 60 . They frequently present as soft, mobile tumors in the superficial extremity or trunk, and tend to be well-differentiated to the extent that they frequently cannot be identified histologically as different from surrounding adipose tissue. Grossly, they appear as soft yellow tissue, with large lobulations, and a very thin capsule.

These tumors rarely occur within the abdomen. If they are found intra-abdominally, they most commonly originate within the colonic wall, though any organ within the alimentary tract can be affected. ${ }^{4}$ Small bowel submucosal lipomas account for only $2.6 \%$ of nonmalignant intestinal tract tumors ${ }^{5}$ and, when found within the small intestine, most commonly occur in the ileum. ${ }^{6}$ Most gastrointestinal lipomas present as solitary masses, though in less than $10 \%$ of cases, multiple lipomas may occur, especially when found within the cecum. ${ }^{7}$

Three pathological subtypes of small bowel lipomas exist: submucosal, intermuscular, and subserosal. Most GI tract lipomas are discovered incidentally in asymptomatic patients. However, lipomas larger than $2 \mathrm{~cm}$ may be the cause of gastrointestinal bleeding or obstruction as they grow into the intestinal lumen. ${ }^{8}$ As in this patient, lipomas may also act as a lead point for intussusception, causing a series of abdominal complaints such as pain, bleeding, and intermittent obstruction. The triad of abdominal pain, currant jelly-like stools, and sausage shaped mass seen in pediatric patients is rarely seen in adults. ${ }^{9}$

Diagnosis of small bowel lipomas is obtained via imaging, with several effective modalities described in the literature. Common imaging modalities used to identify intraluminal lipomas in the bowel are conventional endoscopy, capsule endoscopy, or gastrograffin GI studies. A gastrograffin study is an effective choice, identifying lipomas by revealing the presence of round filling defects with or without a luminal pedicle. Ultrasound may identify intussusception with the characteristic "target sign" finding, and a finding of cleft mass with a clear boundary, low blood supply, and strong ultrasonic echogenicity may indicate a lipoma. ${ }^{10}$ An alternative imaging modality for visualizing these tumors is an upper gastrointestinal (UGI) series with small bowel follow-through. As in this patient, CT is a very commonly used diagnostic study for intussusception, and can also identify lipoma with characteristics of uniform tumor density, clear borders, and fat density. ${ }^{11}$ However, despite the numerous imaging 
techniques available, only $32 \%$ to $50 \%$ of cases of small bowel lipoma are diagnosed preoperatively. ${ }^{12}$

\section{Conclusion}

Small bowel intussusception is a rare adult disease. In most cases, it is caused by benign masses acting as a lead point such as lipomas, polyps, adenomas, Meckel's diverticulum, or inflamed Peyer's patches. When imaging identifies GI intussusception and this is coupled with chronicity of crampy abdominal pain, a diagnostic laparoscopy is warranted. If an intraluminal lead point mass is identified intraop, then resection is necessary for definitive diagnosis and treatment. In this case, a mid-jejunal, subserosal lipoma acting as a lead point for intussusception was clearly visible intra-operatively. The small bowel was safely resected intracoporeally and reanastamosed without complication. The patient has recovered appropriately and has been pain free since surgery with complete remission of aforementioned GI symptoms.

\section{Acknowledgements}

None.

\section{Conflicts of Interest}

The views expressed in this abstract/manuscript are those of the author(s) and do not reflect the official policy or position of the Department of the Army, Department of Defense, or the US Government.

\section{Financial Disclosure}

No financial disclosure to declare.

\section{References}

1. Vizeteu R, Damian M. Intussusception in adult-a rare case. Chirurgia (Bucur). 2011;106(1):119-120.
2. Begos DG, Sandor A, Modin IM. The diagnosis and management of adult intussusception. Am J Surg. 1997;173(2):88-94.

3. Nagorney DM, Sarr MG, McIlrath DC. Surgical management of intussusception in the adult. Ann Surg. 1981;193(2):230-236.

4. Allos Z, Zhubandykova D. Large benign submucosal lipoma presented with descending colonic intussusception in an adult. Am J Case Rep. $2013 ; 14: 245-249$

5. Ackerman NB, Chughtai SQ. Symptomatic lipomas of the gastrointestinal tract. Surg Gynecol Obstet. 1975;141(4):565-568.

6. Akçay MN, Polat M, Cadirci M, et al. Tumor-induced ileo-ileal invagination in adults. American Surgeon. 1994;60(12):980-981.

7. Shehzad KN, Monib S, Ahmad OF, et al. Submucosal lipoma acting as a leading point for colo-colic intussusception in an adult. J Surg Case Rep. 2013; p.10.

8. Kang B, Zhang Q, Shang D1, et al. Resolution of intussusception after spontaneous expulsion of an ileal lipoma per rectum: a case report and literature review. World J Surg Oncol. 2014;12:143.

9. Franc -Law JM, Begin LR, Vasilevsky CA, et al.The dramatic presentation of colonic lipomata: report of two cases and review of the literature. Am Surg. 2001;67(5):491-494.

10. Namikawa $\mathrm{T}$, Hokimoto $\mathrm{N}$, Okabayashi $\mathrm{T}$, et al. Adult ileoileal intussusception induced by an ileal lipoma diagnosed preoperatively: report of a case and review of the literature. Surg Today. 2012;42(7):686692

11. Vagholkar K, Chavan R, Mahadik A, et al.Lipoma of the Small Intestine: A Cause for Intussusception in Adults. Case Reports in Surgery. 2015;2015:856030.

12. Meshikhes AW, Al-Momen SA, Al Talaq FT, et al. Adult intussusception caused by a lipoma in the small bowel: report of a case. Surgery Today. 2005;35(2):161-163. 